\title{
Tacalcitol in the Treatment of Acquired Perforating Collagenosis
}

\author{
J.C. Escribano-Stablé C. Doménech J. Matarredona J.C. Pascual \\ A. Jaen J. Vicente \\ Dermatology Department, Elx/Elche General University Hospital, Elx/Elche, Spain
}

\section{Key Words}

Acquired perforating collagenosis - Perforating collagenosis - Elastosis perforans serpiginosa · Vitamin D3 analogues . Tacalcitol

\begin{abstract}
Acquired perforating collagenosis (APC) is a rare perforating dermatosis characterized by transepidermal collagen elimination. We describe a 65-year-old patient, with long-standing type 2 diabetes mellitus and a 2-year history of itchy hyperkeratotic nodules situated on the back, who was subsequently diagnosed with APC. Treatment included topical corticosteroids and antihistamines, without improvement of the lesions. However, therapy with topical tacalcitol administered for 2 months produced a significant response leading to complete remission of APC.

(c) 2014 S. Karger AG, Basel
\end{abstract}

\section{Introduction}

Acquired perforating collagenosis (APC) is known to be a really difficult disease to treat. The exact etiopathogenesis of this skin disorder has not yet been well established. This fact complicates the development of an effective therapy. To date, several treatments have been used, including corticosteroids, retinoids, antihistamines, antibiotics, allopurinol and phototherapy, with variable success.

\section{Case Report}

We describe a 65-year-old patient with long-standing type 2 diabetes mellitus who presented with a 2-year history of papular lesions about 3-4 $\mathrm{mm}$ in diameter, hyperkeratot- 
ic, very itchy and located primarily on the back (fig. 1). Laboratory tests were unremarkable. A skin biopsy was taken. The hematoxylin-eosin stain showed an ulcerative lesion with collagen fibers, keratinous material and lymphocytic infiltration. The lower edge of the ulcer was interrupted by collagen fibers running perpendicular to it (fig. 2). These findings were consistent with APC. Use of topical corticosteroids and antihistamines did not result in any improvement of the skin lesions.

The lesions were treated for 2 months with topical tacalcitol (once daily) and disappeared completely (fig. 3). However, 4 weeks after finishing the treatment, the lesions recurred less severely, showing an early recovery after reintroduction of tacalcitol during 3 weeks. Now, the patient is treating himself when the lesions recur using the same treatment with full recovery between outbreaks.

\section{Discussion}

Perforating skin diseases are characterized by hyperkeratotic papular lesions where the connective tissue fibers are eliminated through the epidermis. Different clinical types can be found within this group of skin disorders. Reactive perforating collagenosis usually appears during childhood, following a history of trauma to the skin. The appearance of collagen fibers across the epidermis is characteristic in histological studies. Elastosis perforans serpiginosa typically develops in children and young adults. In up to $40 \%$ of cases, it is related to genetic disorders (Ehlers-Danlos syndrome, Down syndrome, acrogeria and others). Histologically, elastin fibers perforate the epidermis. Both dermatoses share a hereditary basis, with a scarce prevalence in the general population. APC tends to occur in adults, usually related to diseases with a long-term outcome such as chronic renal failure or diabetes mellitus and less frequently in chronic hepatopathy, hematologic malignancies and solid neoplasia. Finally, there is a wide variety of perforating dermatoses called secondary dermatosis, in which transepidermal elimination of connective tissue fibers occurs secondary to other basic dermatological conditions such as mycotic infections, granulomas or skin neoplasm [1-3].

An accurate etiopathogenesis of APC has not been determined. Patients with type 2 diabetes mellitus and chronic kidney failure, mainly those treated with hemodialysis, are known to develop microvascular diseases leading to intense pruritus. Repeated microtrauma caused by scratching may cause necrosis in the connective tissue fibers of the dermis, resulting in transepidermal elimination [4, 5]. Moreover, these patients show epithelial cell hyperplasia, motivating an inflammatory foreign body reaction to altered collagen and elastin fibers. Levels of fibronectin are increased in the plasma and skin where perforating lesions appear in patients with diabetes and uremia. Fibronectin has been proven to have an influence on cell differentiation and migration. In the case of perforating dermatoses, fibronectin is associated with type IV collagen fibers and keratinocytes causing epithelial cell hyperplasia, therefore triggering an onset of perforating injuries [6].

With regard to its treatment, there is a clear correlation between the disappearance of pruritus and improvement of the lesion. Antihistamines and corticosteroids are occasionally helpful in the treatment of APC. Other alternative therapies have been tested, although experience is summarized in a small series of cases. Allopurinol has been used, regardless of plasma levels of uric acid, with good results. Its effectiveness seems to be related to its antioxidant effect, since it is capable of inhibiting xanthine oxidase, thus decreasing the production of oxygen free radicals that damage collagen fibers, and collagen fiber crosslinking itself [7]. 
Escribano-Stablé et al.: Tacalcitol in the Treatment of Acquired Perforating Collagenosis

Narrowband ultraviolet B has also been used in the treatment of APC. Apart from an antiproliferative and anti-inflammatory effect, this therapy seems to relieve the pruritus, consequently improving lesions, although recurrence happens in a variable interval once narrowband ultraviolet B treatment is stopped [8].

In some cases, antimicrobials such as rifampicin, clindamycin and doxycycline have been used, with an improvement in lesions. This seems to prove that the presence of infectious agents may contribute to the development of APC [9-11].

Tazarotene is a topical retinoid of selective action on retinoic acid receptors. Its main action mechanism is through the regulation of keratin, with a significant antiproliferative effect. It is used primarily in the treatment of plaque psoriasis, although various cases have been published where treatment with tazarotene provides an improvement of the perforating collagenosis lesions [12].

Finally, tacalcitol is a vitamin D3 synthetic analogue used in the topical treatment of psoriasis. This drug inhibits the proliferation of keratinocytes and, at the same time, modulates inflammatory mediators and the skin's immune system. No previous data has been published on the use of tacalcitol in perforating collagenosis. As mentioned above, APC lesions are hyperkeratotic with a significant inflammatory reaction with epithelial cell hyperplasia. That is, similar phenomena to those in psoriasis [13]. On this basis, we decided to use this drug in the treatment of our patient, achieving excellent results.

\section{Disclosure Statement}

The authors declare no conflicts of interest.

\section{References}

1 Tsuboi H, Katsuoka K: Characteristics of acquired reactive perforating collagenosis. J Dermatol 2007;34:640-644.

-2 Faver IR, Daoud MS, Su WP: Acquired perforating collagenosis. Report of six cases and review of the literature. J Am Acad Dermatol 1994;30:575-580.

-3 Saray Y, Seçkin D, Bilezikçi B: Acquired perforating dermatosis: clinicopathological features in twenty-two cases. J Eur Acad Dermatol Venereol 2006;20:679-688.

4 Hong SB, Park JH, Ihm CG, et al: Acquired perforating dermatosis in patients with chronic renal failure and diabetes mellitus. J Korean Med Sci 2004;19:283-288.

5 Thiele-Ochel S, Schneider LA: Acquired perforating collagenosis: is it due to damage by scratching? Br J Dermatol 2001;145:173-174.

6 Bileziky B, Seckin D, Demirhan B: Acquired perforating dermatosis in patients with chronic renal failure: a possible role for fibronectin. J Eur Acad Dermatol Venereol 2003;17:230-232.

7 Hoque SR, Ameen M, Holden CA: Acquired reactive perforating collagenosis: four patients with a giant variant treated with allopurinol. Br J Dermatol 2006;154:759-762.

8 Ohe S, Danno K, Sasaki H, et al: Treatment of acquired perforating dermatosis with narrowband ultraviolet B. J Am Acad Dermatol 2004;892-894.

-9 Skiba G, Milkiewicz P, Mutimer D, et al: Successful treatment of acquired perforating dermatosis with rifampicin in an Asian patient with sclerosing cholangitis. Liver 1999;19:160-163.

10 Kasiakou SK, Peppas G, Kapaskelis AM, et al: Regression of skin lesions of Kyrle's disease with clindamycin: implication for an infectious component in the etiology of the disease. J Infect 2005;50:412-416.

11 Gönül M, Cakmak SK, Gül U, et al: Two cases of acquired perforating dermatosis treated with doxycycline therapy. Int J Dermatol 2006;45:1461-1463.

12 Outland JD, Brown TS, Callen JP: Tazarotene is an effective therapy for elastosis perforans serpiginosa. Arch Dermatol 2002;138:169-171.

13 Leone G, Pacifico A: Profile of clinical efficacy and safety of topical tacalcitol. Acta Biomed 2005;76:13-19. 


\section{Case Reports in Dermatology}

Case Rep Dermatol 2014;6:69-73

DOI: $10.1159 / 000360390$

Escribano-Stable et al.: Tacalcitol in the Treatment of Acquired Perforating
Collagenosis

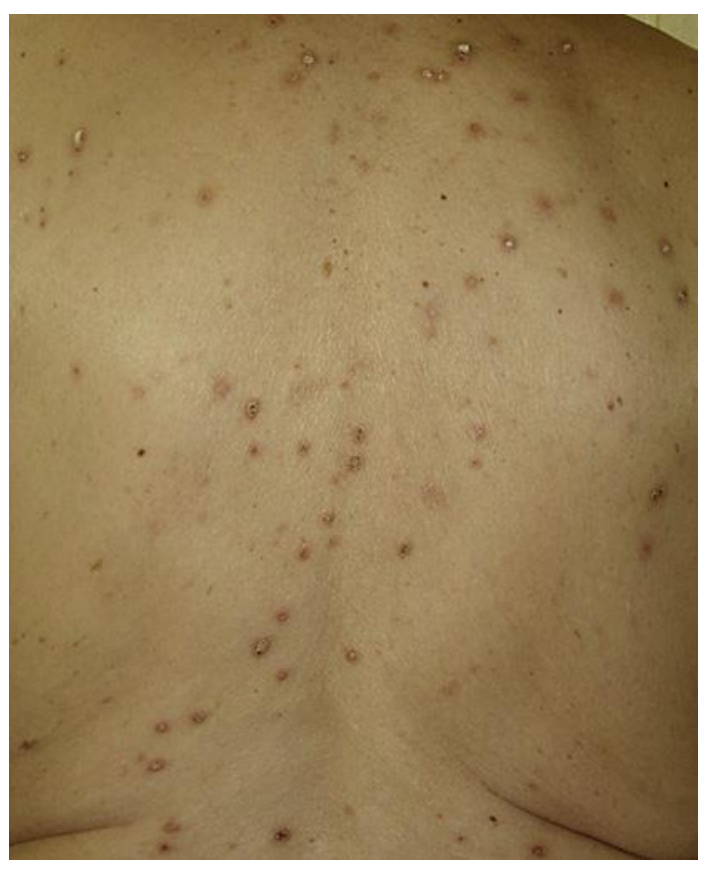

Fig. 1. Papular hyperkeratotic lesions on the patient's back prior to treatment.

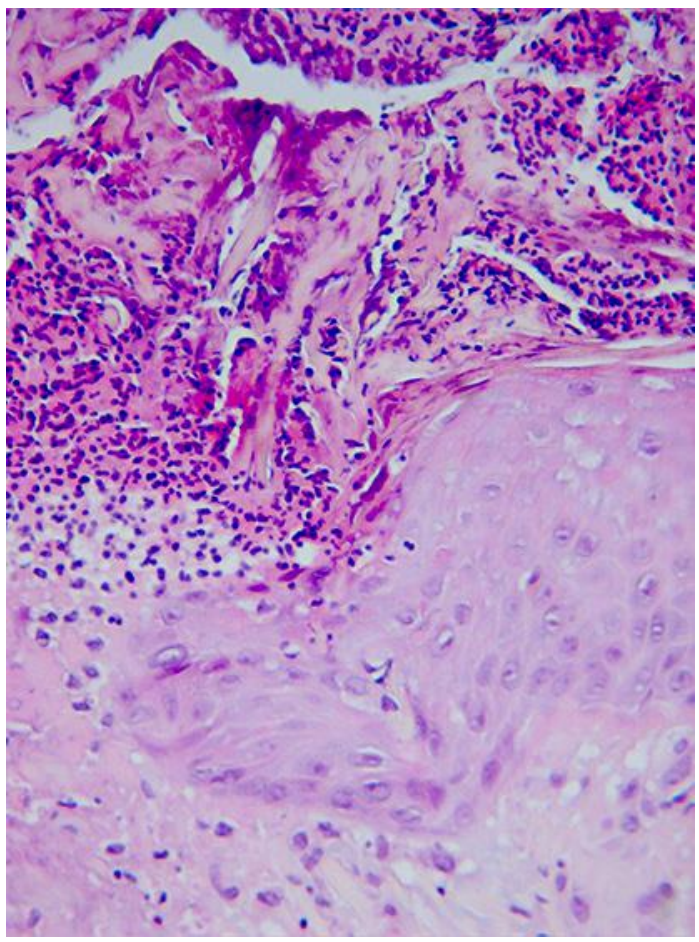

Fig. 2. Ulcer with cell detritus on the top and collagen bundles and elastic fibers on the bottom. 


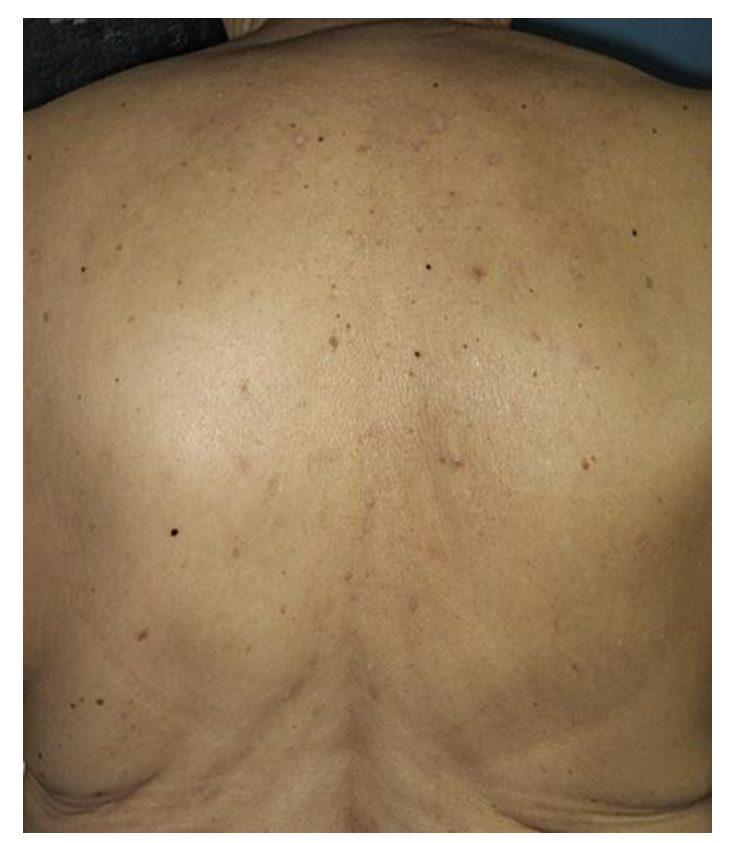

Fig. 3. Complete remission of the skin lesions after 2 months of treatment with topical tacalcitol. 